\title{
Stress related disorders and risk of cardiovascular disease: population based, sibling controlled cohort study
}

Check for updates

For numbered affiliations see end of the article.

Correspondence to: H Song

huan@hi.is or

huan.song@ki.se

(ORCID 0000-0003-3845-8079)

Additional material is published online only. To view please visit the journal online.

Cite this as: BMJ 2019;365:l1255 http://dx.doi.org/10.1136/bmj.l1255

Accepted: 12 March 2019

\author{
Huan Song, ${ }^{1,2}$ Fang Fang, ${ }^{2}$ Filip K Arnberg, ${ }^{3,4}$ David Mataix-Cols, ${ }^{5,6}$ \\ Lorena Fernández de la Cruz, ${ }^{5,6}$ Catarina Almqvist, ${ }^{2,7}$ Katja Fall, ${ }^{2,8}$ Paul Lichtenstein, ${ }^{2}$ \\ Gudmundur Thorgeirsson, ${ }^{1}$ Unnur A Valdimarsdóttir ${ }^{1,2,9}$
}

\section{ABSTRACT}

OBJECTIVE

To assess the association between stress related disorders and subsequent risk of cardiovascular disease.

\section{DESIGN}

Population based, sibling controlled cohort study.

SETTING

Population of Sweden.

\section{PARTICIPANTS}

136637 patients in the Swedish National Patient Register with stress related disorders, including post-traumatic stress disorder (PTSD), acute stress reaction, adjustment disorder, and other stress reactions, from 1987 to 2013; 171314 unaffected full siblings of these patients; and 1366370 matched unexposed people from the general population.

\section{MAIN OUTCOME MEASURES}

Primary diagnosis of incident cardiovascular disease-any or specific subtypes (ischaemic heart disease, cerebrovascular disease, emboli/ thrombosis, hypertensive diseases, heart failure, arrhythmia/conduction disorder, and fatal cardiovascular disease)-and 16 individual diagnoses of cardiovascular disease. Hazard ratios for cardiovascular disease were derived from Cox models, after controlling for multiple confounders.

RESULTS

During up to 27 years of follow-up, the crude incidence rate of any cardiovascular disease was $10.5,8.4$, and 6.9 per 1000 person years among exposed patients,

\section{WHAT IS ALREADY KNOWN ON THIS TOPIC}

Accumulating evidence suggests a role of severe stress reactions to significant life events or trauma in cardiovascular disease

Most evidence comes from studies of male samples (veterans or active duty military personnel) focusing on post-traumatic stress disorder (PTSD) or self reported PTSD symptoms

The modest sample sizes of previous studies mean that limited data exist on potential differences in immediate and long term effects of stress reactions on multiple types of cardiovascular disease

\section{WHAT THIS STUDY ADDS}

Severe stress reactions to significant life events or trauma are associated with considerably elevated risk of multiple types of cardiovascular disease

This is especially the case during the first year after the diagnosis of a stress related disorder

The association between stress related disorders and cardiovascular disease seems particularly strong in cases of early onset diseases (occurring before the age of 50) their unaffected full siblings, and the matched unexposed individuals, respectively. In sibling based comparisons, the hazard ratio for any cardiovascular disease was 1.64 (95\% confidence interval 1.45 to 1.84$)$, with the highest subtype specific hazard ratio observed for heart failure $(6.95,1.88$ to 25.68$)$, during the first year after the diagnosis of any stress related disorder. Beyond one year, the hazard ratios became lower (overall 1.29, 1.24 to 1.34), ranging from 1.12 (1.04 to 1.21) for arrhythmia to 2.02 (1.45 to 2.82) for artery thrombosis/embolus. Stress related disorders were more strongly associated with early onset cardiovascular diseases (hazard ratio 1.40 (1.32 to 1.49$)$ for attained age $<50)$ than later onset ones (1.24 (1.18 to 1.30) for attained age $\geq 50$; $P$ for difference $=0.002$ ). Except for fatal cardiovascular diseases, these associations were not modified by the presence of psychiatric comorbidity. Analyses within the population matched cohort yielded similar results (hazard ratio 1.71 (1.59 to 1.83) for any cardiovascular disease during the first year of followup and 1.36 (1.33 to 1.39) thereafter).

\section{CONCLUSION}

Stress related disorders are robustly associated with multiple types of cardiovascular disease, independently of familial background, history of somatic/psychiatric diseases, and psychiatric comorbidity.

\section{Introduction}

Most people are, at some point during their life, exposed to psychological trauma or stressful life events such as the death of a loved one, a diagnosis of life threatening illness, natural disasters, or violence. ${ }^{12}$ Accumulating evidence suggests that such adversities might lead to an increased risk of several major diseases (including cardiovascular morbidity, injury, infection, and certain autoimmune diseases but not cancer) and mortality, ${ }^{3-10}$ with the largest risk elevations usually noted among people who develop psychiatric disorders as a result of their trauma. ${ }^{11-13}$

Stress related disorders are a group of psychiatric disorders for which one of the diagnostic criteria is the presence of a preceding stressful life event. Depending on the type of stressor, the reported symptoms, and their duration, such disorders are mainly categorised as acute stress reaction, post-traumatic stress disorder (PTSD), and adjustment disorder. ${ }^{14}$ The presence of a life threatening traumatic event is a prerequisite for the former two disorders, whereas adjustment disorder generally refers to physical or psychological distress ("adjustment syndromes"15) 
triggered by an identifiable and significant life change. PTSD is the most severe and widely studied form of stress related disorder, characterised by reexperiencing, avoidance, negative cognitions and mood, and hyperarousal following the traumatic event. ${ }^{16}$ We summarised the existing evidence on PTSD and cardiovascular consequences from 11 prospective cohort investigations (see supplementary table A), all of which suggested an elevated incidence of cardiovascular diseases among people with PTSD. However, owing to sample size restrictions of previous studies, the evidence for the role of PTSD in specific types of cardiovascular disease is still limited. Furthermore, the potential role of other stress related disorders-acute stress reaction, adjustment disorder, and other stress reactions-in the development of cardiovascular disease remains largely unexplored. Finally, cardiovascular diseases tend to have a genetic predisposition, as well as risk factors related to lifestyle and comorbidities that may cluster within families and exert an as yet unknown influence on the association between stress related disorders and cardiovascular disease. ${ }^{17-19}$

Taking advantage of the nationwide population and health registers in Sweden, which provide virtually complete information on all medical diagnoses and family links, we did a population based sibling analysis and a matched cohort study to elucidate the role of stress related disorders in the development of cardiovascular disease while controlling for familial confounders, history of psychiatric and somatic conditions, and psychiatric comorbidities.

\section{Methods}

\section{Study design}

The Swedish personal identification number uniquely assigned to all residents in Sweden at birth or immigration enables data linkage across several nationwide registers in Sweden. ${ }^{20}$ We first identified an exposed cohort of all people born in Sweden who received their first diagnosis of a stress related disorder between 1 January 1987 and 31 December 2013 ( $n=156537$; fig 1) from the Swedish National Patient Register. The National Patient Register has nationwide information on inpatient care since 1987 and on hospital based outpatient specialist care since 2001 (with initial coverage of $>80 \%$ ). We excluded patients who received a diagnosis before age $5(n=139),{ }^{21}$ had a history of any cardiovascular disease before the diagnosis of a stress related disorder $(n=15899)$, or had conflicting information (died or emigrated before the diagnosis of a stress related disorder; $n=24$ ). To enable the complete familial links from the Swedish MultiGeneration Register that includes largely complete familial information for people born in Sweden from 1932 onward, we excluded 3838 people born before 1932, leaving 136637 patients in the analysis. We considered patients with stress related disorders to be exposed from the date of their diagnosis.

\section{Sibling cohort}

To control for the familial components, ${ }^{22}$ we identified 106180 clusters of full siblings discordant for stress related disorders with a total of 171314 unaffected full siblings who were alive and free of stress related disorders and cardiovascular disease at the diagnosis

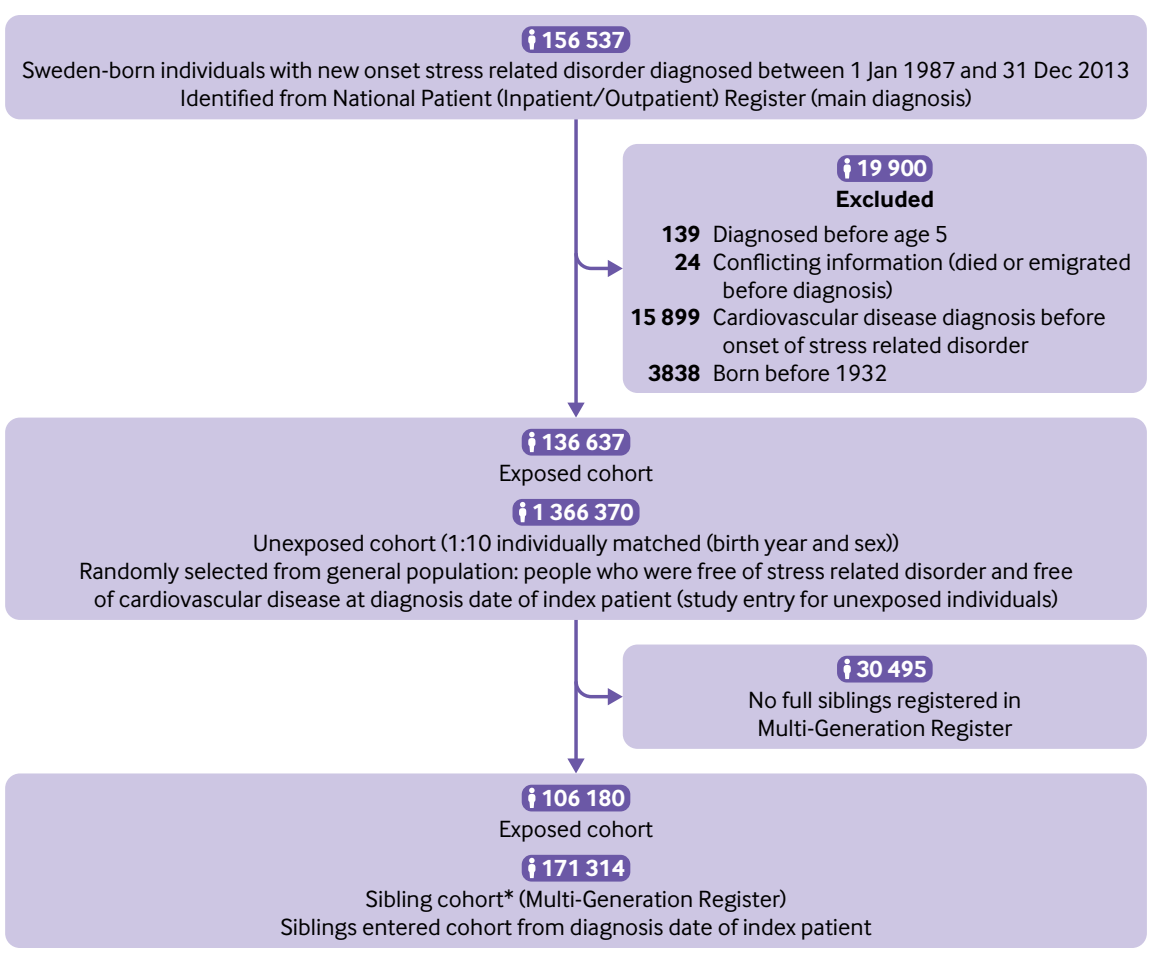

Fig 1 | Study design. CVD=cardiovascular disease. $* 4332$ families had more than one identified case of stress related disorder 
date of the affected sibling, through the MultiGeneration Register. We used the date of diagnosis of the affected sibling as the index date for both siblings.

\section{Population matched cohort}

We compared the patients with stress related disorders with the general population in a matched cohort. For each exposed patient, we randomly selected 10 people from the Total Population Register who were free of stress related disorders and cardiovascular disease at the diagnosis date of the index patient. Exposed patients and unexposed people were individually matched by birth year and sex. We used the date of diagnosis as the index date for both exposed patients and the matched individuals.

\section{Follow-up}

Follow-up of all study participants was from the index date until the first primary diagnosis of cardiovascular disease (any or specific subtype), death, emigration, or the end of follow-up (31 December 2013), whichever occurred first. The follow-up time for the unaffected full siblings or matched unexposed people was additionally censored at the time of their first diagnosis of stress related disorder, if any, during the follow-up.

\section{Definition of stress related disorders}

We defined stress related disorders as any first outpatient or inpatient visit with the main diagnosis of the 9th or 10th Swedish revisions of the international classification of diseases (ICD) codes 308 or 309 (ICD9) or F43 (ICD-10), according to the National Patient Register. We further divided stress related disorders into PTSD (ICD-9: 309B; ICD-10: F43.1), acute stress reaction (ICD-9: 308, 309A; ICD-10: F43.0), and adjustment disorder and other stress reactions (ICD9: 309X; ICD-10: F43.8, F43.9). Because PTSD may initially be preceded by acute stress reaction or other stress related disorders, ${ }^{23}$ we considered all patients who received a diagnosis of PTSD within one year after their first stress related disorder diagnosis to be PTSD patients.

\section{Definition of cardiovascular diseases}

We defined an incident cardiovascular disease event (any; specific subtypes including ischaemic heart disease, cerebrovascular disease, emboli/ thrombosis, hypertensive disease, heart failure, and arrhythmia/conduction disorder; or individual events) as an outpatient or inpatient visit (according to the National Patient Register) with a main diagnosis of cardiovascular disease or as a death (according to the Cause of Death Register) with cardiovascular disease as the underlying cause, using corresponding ICD codes (see supplementary table B). We defined a fatal cardiovascular disease as death within 30 days after an incident cardiovascular disease event. ${ }^{24}$

\section{Covariates}

Stress related disorders show substantial comorbidity with other psychiatric disorders, such as mood and anxiety disorders. ${ }^{25}$ We therefore defined other psychiatric disorders as "history of other psychiatric disorders" if the first diagnosis was made more than three months before the diagnosis of a stress related disorder. We defined other psychiatric disorders that had a first diagnosis made within three months before and one year after the diagnosis of a stress related disorder as "psychiatric comorbidity." Diagnoses of other psychiatric disorders also came from the National Patient Register (ICD-8: 290-319 except 307, 308.4; ICD-9: 290-319 except 308, 309; and ICD-10: F10-F99 except F43).

We retrieved information about educational level, family income, and marital status from the Longitudinal Integration Database for Health Insurance and Labor Market. History of severe somatic diseases, including chronic pulmonary disease, connective tissue disease, diabetes, renal diseases, liver diseases, ulcer diseases, and HIV infection/AIDS, which are considered to contribute to survival time, ${ }^{26}$ were collected on the basis of the National Patient Register (ICD codes listed in supplementary table B). We defined family history of cardiovascular disease as any cardiovascular event among any first degree relatives (that is, biological parents and full siblings) of the participants according to the National Patient Register and the Cause of Death Register. In all analyses, we used the most recent information before the index date for each covariate, except for psychiatric comorbidity (as defined above).

\section{Statistical analysis}

We first visualised the time dependent associations of stress related disorders with the risk of cardiovascular disease by using flexible parametric survival models. ${ }^{27}$ Because of the greatly increased risk of cardiovascular disease during the time period immediately after the diagnosis of stress related disorders, compared with thereafter, we assessed the associations separately during the first year after a diagnosis of stress related disorder and beyond this one year period $(<1$ or $\geq 1$ year of follow-up), using hazard ratios with $95 \%$ confidence intervals derived from conditional Cox regression models. We used time since the index date as the underlying time scale.

In the sibling cohort, we stratified all analyses by family identifiers and partially (in simply adjusted models) or fully (in fully adjusted models) adjusted for multiple potential confounders, including age at index date, sex, educational level ( $<9$ years, 9-12 years, $>12$ years, or unknown), family income (top $20 \%$, middle, lowest $20 \%$, or unknown), marital status (single, married/cohabiting, or divorced/widowed), history of severe somatic diseases (yes or no), and history of psychiatric disorders (yes or no). We considered all stress related disorders as one group and also did separate analyses for PTSD, acute stress reaction, and adjustment disorder and other stress reactions. In subgroup analyses, we calculated the hazard ratios by sex (male or female), age at index date (by thirds: $\leq 28$, $29-42$, or $\geq 43$ years), attained age (age during followup: $<50$ or $\geq 50$ years), calendar year at index date 
(1987-96, 1997-2006, or 2007-13), follow-up period ( $<1$ year, 1-4 years, 5-9 years, or $\geq 10$ years), family history of cardiovascular disease (yes or no), history of severe somatic diseases (yes or no), and history of other psychiatric disorders (yes or no).

In addition to any cardiovascular disease, we examined the hazard ratios for seven major categories (including fatal cardiovascular diseases) and 16 individual diagnoses of cardiovascular disease. We further analysed certain acute cardiovascular events (cardiac arrest, acute myocardial infarction, and acute cerebrovascular disease) as a hypothesised group of immediate cardiac consequences triggered by a stress related disorder, and therefore with more specific time intervals during the first year of follow-up ( $<1$ month, 1-5 months, 6-11 months, 1-4 years, 5-9 years, and $\geq 10$ years).

To study the potential role of psychiatric comorbidity in the studied associations, we did further subgroup analyses by the presence of psychiatric comorbidity. Within one year after the diagnosis of a stress related disorder, we considered psychiatric comorbidity as a time varying variable (that is, the status of psychiatric comorbidity changed from "no" to "yes" at the date of diagnosis of the psychiatric comorbidity).

We did similar analyses in the population matched cohort, in which we used Cox regression models stratified by matching identifiers (birth year and sex) and adjusted for all above mentioned covariates and family history of cardiovascular disease (yes/no). Additionally, to examine the robustness of the observed estimates to the definition of history of psychiatric disorders, we re-ran the analyses by using a six month, instead of three month, threshold before the diagnosis of a stress related disorder, to distinguish "history of psychiatric disorders" from "psychiatric comorbidity." We used SAS statistical software, version 9.4 and Stata 15 for all analyses.

\section{Patient and public involvement}

No patients were involved in setting the research question or the outcome measures, nor were they involved in developing plans for design or implementation of the study. A patient was invited to contribute to the review process of the study, and we are grateful for his input on the readability and accuracy of this document. There are no plans to directly disseminate the results of the research to study participants or the relevant patient community. The dissemination to the Swedish population (which constitutes the study population) will be achieved through media outreach (for example, press release and communication) on publication of this study.

\section{Results}

The median follow-up time was 6.2, 6.9, and 6.5 years for exposed patients, unaffected full siblings, and matched unexposed people, respectively, accumulating 2268901 person years at risk in the sibling cohort and 12001887 person years in the population matched cohort (table 1). Median age at index date was 36 years. Most (63\%) people exposed to stress related disorders were women, whereas the unaffected siblings had an equal sex distribution. Having a history of other psychiatric disorders was more common among exposed patients in both cohorts (34-35\%) than among the unaffected siblings (12\%), as well as among the matched unexposed individuals $(8 \%)$. In addition, patients with stress related disorders tended to have a higher burden of somatic diseases at the index date and a lower family income level, and to be more likely to be divorced or widowed, compared with their unaffected siblings or matched unexposed people.

Using flexible parametric models, we observed a peak of cardiovascular disease risk immediately after diagnosis of a stress related disorder, followed by a rapid decline within the first six months (supplementary figure A). After one year, the magnitude of the hazard ratios tended to be constant (around 1.3 for both sibling based and population based comparisons). During up to 27 years of follow-up, the crude incidence rate of any cardiovascular disease was $10.5,8.4$, and 6.9 per 1000 person years

Within the first year of follow-up, 1617 people in the sibling cohort had a cardiovascular event (table 2 , left side). The incidence rate among the exposed patients was almost twice that of their full siblings (crude: $8.1 v 4.9 / 1000$ person years; age standardised: $8.7 v 4.5 / 1000$ person years). With a total number of 18522 cardiovascular events identified beyond one year of follow-up, the corresponding incidence rates were $10.3 v 8.9 / 1000$ person years (age standardised: $6.0 v 4.5 / 1000$ person years) for exposed patients and their full siblings. When we added history of other psychiatric disorders and history of severe somatic diseases to the Cox models, the obtained hazard ratios decreased from 1.77 (95\% confidence interval 1.58 to 1.98 ) to 1.64 (1.45 to 1.84 ) for a less than one year period and from 1.39 (1.34 to 1.44 ) to 1.29 (1.24 to 1.34) for one year or longer. The main attenuation of hazard ratios occurred after additional adjustment for history of other psychiatric disorders. We obtained similar findings when comparing exposed patients with matched unexposed people (table 2, right side), with seemingly larger attenuation of hazard ratios after full adjustment for multiple risk factors, compared with the sibling analysis. Furthermore, although the largest point estimates were observed for PTSD, the subgroup analyses showed comparable hazard ratios for other types of stress related disorders (supplementary tables C-E).

We observed similar associations across sex, calendar period, history of severe somatic diseases, history of psychiatric disorders, and family history of cardiovascular disease (table 3 and supplementary table F) but stronger associations among people with younger age at index date (sibling analysis: hazard ratio 1.52 (1.35 to 1.71 ), 1.26 (1.17 to 1.36 ), and 1.30 (1.24 to 1.37 ) for age $\leq 28,29-41$, and $\geq 42$ years, respectively; $\mathrm{P}$ for interaction=0.010). Additionally, we observed a stronger association between stress 
Table 1 | Characteristics of study cohorts. Values are numbers (percentages) unless stated otherwise

\begin{tabular}{|c|c|c|c|c|}
\hline \multirow[b]{2}{*}{ Characteristics } & \multicolumn{2}{|l|}{ Sibling cohort } & \multicolumn{2}{|c|}{ Population matched cohort } \\
\hline & $\begin{array}{l}\text { Exposed patients } \\
(\mathrm{n}=106180)\end{array}$ & $\begin{array}{l}\text { Unaffected full siblings } \\
(n=171314)\end{array}$ & $\begin{array}{l}\text { Exposed patients } \\
(\mathrm{n}=136637)\end{array}$ & $\begin{array}{l}\text { Matched unexposed individuals } \\
(n=1366370)\end{array}$ \\
\hline Median (IQR) age at index date, years & $36(25-46)$ & $36(25-47)$ & $35(24-46)$ & $35(24-46)$ \\
\hline Median (IQR) follow-up time, years & $6.2(2.8-10.3)$ & $6.9(3.3-11.6)$ & $6.2(2.8-10.4)$ & $6.5(3.0-10.8)$ \\
\hline Male sex & $39821(37.50)$ & $86751(50.64)$ & $51251(37.51)$ & $512510(37.51)$ \\
\hline \multicolumn{5}{|l|}{ Educational level: } \\
\hline$<9$ years & $3780(3.56)$ & $9270(5.41)$ & $5207(3.81)$ & $45425(3.32)$ \\
\hline $9-12$ years & $75863(71.45)$ & $118617(69.24)$ & $98295(71.94)$ & $891626(65.26)$ \\
\hline$>12$ years & $24771(23.33)$ & $38467(22.45)$ & $30570(22.37)$ & $402935(29.49)$ \\
\hline Unknown & $1766(1.66)$ & $4960(2.90)$ & $2565(1.88)$ & $26384(1.93)$ \\
\hline \multicolumn{5}{|l|}{ Yearly family income level: } \\
\hline Lowest $20 \%$ & $24351(22.93)$ & $32905(19.21)$ & $31919(23.36)$ & $239434(17.52)$ \\
\hline Middle & $58081(54.70)$ & $87383(51.01)$ & $73752(53.98)$ & $751115(54.97)$ \\
\hline Top 20\% & $13014(12.26)$ & 25854 (15.09) & $16422(12.02)$ & $226400(16.57)$ \\
\hline Unknown & $10734(10.11)$ & $25172(14.69)$ & $14544(10.64)$ & $149421(10.94)$ \\
\hline \multicolumn{5}{|l|}{ Marital status,: } \\
\hline Single & $62369(58.74)$ & $98676(57.60)$ & $80714(59.07)$ & $809763(59.26)$ \\
\hline Married or cohabiting & $30435(28.66)$ & $58005(33.86)$ & $38545(28.21)$ & $461819(33.80)$ \\
\hline Divorced or widowed & $13376(12.60)$ & $14633(8.54)$ & $17378(12.72)$ & $94788(6.94)$ \\
\hline History of severe somatic diseases* & $18929(17.83)$ & $24180(14.11)$ & $25133(18.39)$ & $168825(12.36)$ \\
\hline History of other psychiatric disordert & $36559(34.43)$ & $21117(12.33)$ & $47962(35.10)$ & $106160(7.77)$ \\
\hline Family history of cardiovascular disease & $51969(48.94) \neq$ & 84257 (49.18)‡ & $64448(47.17)$ & $604782(44.26)$ \\
\hline \multicolumn{5}{|l|}{ Type of stress related disorder: } \\
\hline Post-traumatic stress disorder & $8660(8.16)$ & - & $11162(8.17)$ & - \\
\hline Acute stress reaction & $48804(45.96)$ & - & $63058(46.15)$ & - \\
\hline $\begin{array}{l}\text { Adjustment disorder and other stress } \\
\text { reactions }\end{array}$ & $48716(45.88)$ & - & $62417(45.68)$ & - \\
\hline Psychiatric comorbidity§ & $18893(17.79)$ & $2811(1.64)$ ๆ & $24169(17.69)$ & $12963(0.95) \emptyset$ \\
\hline \multicolumn{5}{|c|}{$\begin{array}{l}\text { IQR=interquartile range. } \\
\text { *Includes chronic pulmonary disease, connective tissue disease, diabetes, renal diseases, liver diseases, ulcer diseases, and HIV infection/AIDS. } \\
\text { tFirst diagnosis of a psychiatric disorder, other than stress related disorders, occurred }>3 \text { months before index date. } \\
\text { ¥Marginal difference between exposed and unexposed sibling group is due to different number of siblings for exposed patients. Within each family, family history of cardiovascular disease is } \\
\text { always the same. } \\
\text { \$New onset psychiatric disorder, other than stress related disorders, that was diagnosed between } 3 \text { months before and } 1 \text { year after diagnosis of studied stress related disorder. } \\
\text { १For unaffected full siblings and matched unexposed individuals, number and percentage of individuals who had diagnosis of other psychiatric disorder between } 3 \text { months and } 1 \text { one year after } \\
\text { index date. }\end{array}$} \\
\hline
\end{tabular}

related disorders and early onset cardiovascular diseases (sibling analysis: hazard ratio 1.40 (1.32 to 1.49) for attained age $<50$ years) than later onset ones (1.24 (1.18 to 1.30 ) for attained age $\geq 50$ years) ( $P$ for difference=0.002).

Figure 2 shows increased hazard ratios for all studied categories of cardiovascular diseases (including fatal cardiovascular disease events) and for most individual diagnoses of cardiovascular diseases in patients with stress related disorders compared with their unaffected full siblings. The highest hazard ratios were for heart failure $(6.95,1.88$ to 25.68), other cerebrovascular disease $(5.64,1.19$ to 26.75$)$, conduction disorder (5.00, 1.58 to 15.80$)$, and cardiac arrest (3.37, 1.05 to 10.75$)$ during the first year after the diagnosis of a stress related disorder. Beyond one year, the relative risk elevations were attenuated (hazard ratios ranged from 1.12 (1.04 to 1.21) for

\begin{tabular}{|c|c|c|c|c|}
\hline \multirow[b]{2}{*}{ Model information } & \multicolumn{2}{|l|}{ Sibling cohort } & \multicolumn{2}{|l|}{ Population matched cohort } \\
\hline & $\begin{array}{l}\text { No of cases (incidence*) in } \\
\text { patients/siblings }\end{array}$ & $\begin{array}{l}\text { Hazard ratio }(95 \% \\
\mathrm{Cl}) \dagger\end{array}$ & $\begin{array}{l}\text { No of cases (incidence*) in } \\
\text { patients/unexposed individuals }\end{array}$ & $\begin{array}{l}\text { Hazard ratio } \\
(95 \% \mathrm{Cl}) \dagger\end{array}$ \\
\hline \multicolumn{5}{|l|}{$\ll 1$ year of follow-up } \\
\hline $\begin{array}{l}\text { Controlled for sex, birth year, educational level, family } \\
\text { income, and marital status }\end{array}$ & \multirow{4}{*}{$811(8.06) / 806(4.92)$} & 1.77 (1.58 to 1.98$)$ & \multirow{4}{*}{$1094(8.45) / 5624(4.32)$} & $1.92(1.80$ to 2.05$)$ \\
\hline As above + history of other psychiatric disorder & & $1.65(1.47$ to 1.86$)$ & & $1.74(1.62$ to 1.86$)$ \\
\hline As above + history of severe somatic diseases & & $1.64(1.45$ to 1.84$)$ & & $1.72(1.60$ to 1.84$)$ \\
\hline As above + family history of cardiovascular disease & & - & & $1.71(1.59$ to 1.83$)$ \\
\hline \multicolumn{5}{|l|}{$\geq 1$ year of follow-up } \\
\hline $\begin{array}{l}\text { Controlled for sex, birth year, educational level, family } \\
\text { income, and marital status }\end{array}$ & \multirow{4}{*}{$7246(10.30) / 11276(8.87)$} & $1.39(1.34$ to 1.44$)$ & \multirow{4}{*}{$9827(10.81) / 69483(7.26)$} & $1.52(1.49$ to 1.56$)$ \\
\hline As above + history of other psychiatric disorder & & $1.30(1.25$ to 1.34$)$ & & $1.39(1.36$ to 1.42$)$ \\
\hline As above + history of severe somatic diseases & & $1.29(1.24$ to 1.34$)$ & & $1.37(1.33$ to 1.40$)$ \\
\hline As above + family history of cardiovascular disease & & - & & $1.36(1.33$ to 1.39$)$ \\
\hline
\end{tabular}

*Per 1000 person years.

tDerived from Cox regression models, stratified by family identifier (for sibling based comparison) or matching identifier (birth year and sex, for population based comparison) and adjusted for covariates listed in model information column. Time since index date was used as underlying time scale. 


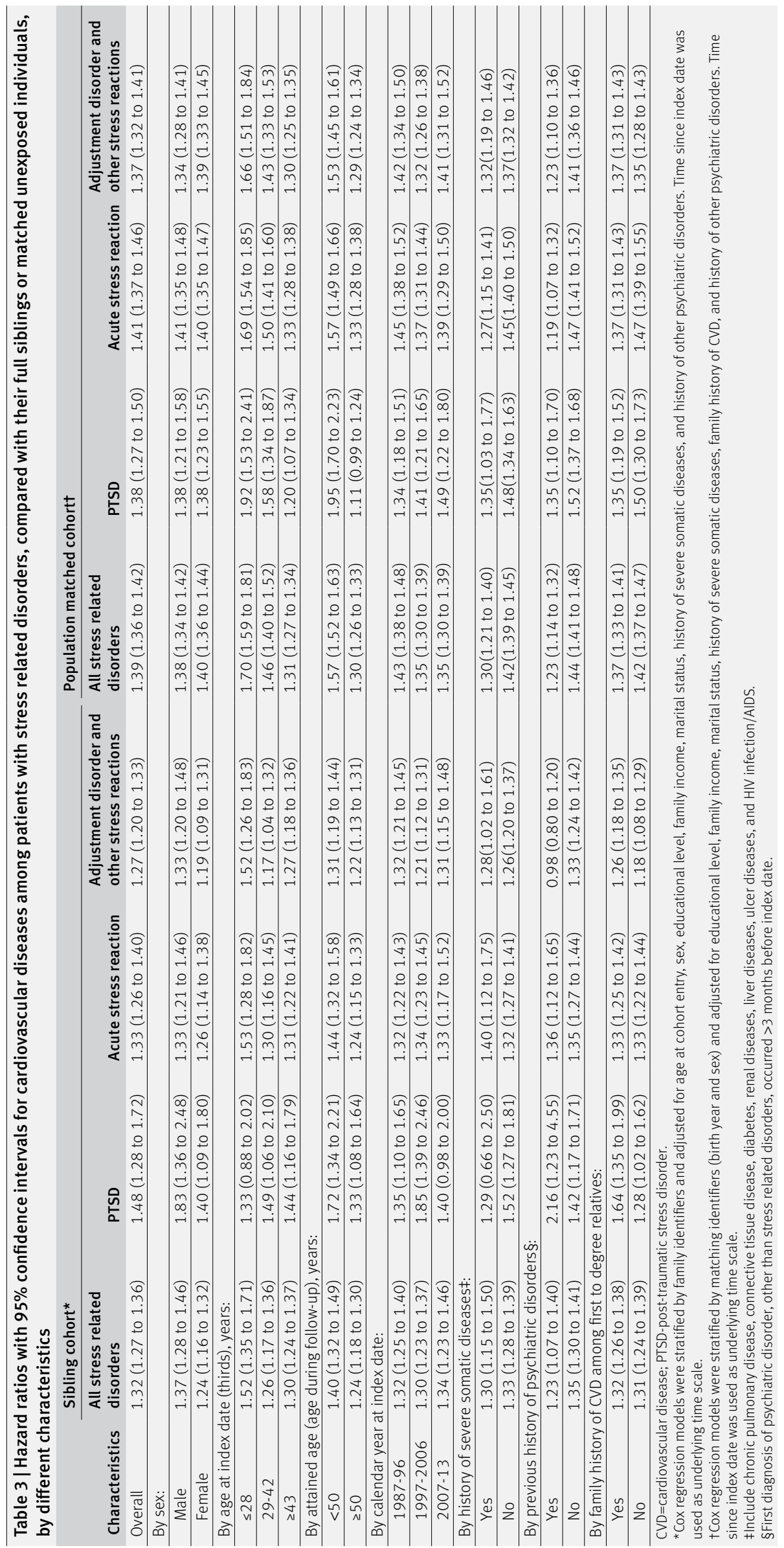




\begin{tabular}{|c|c|c|c|c|c|c|}
\hline \multirow[b]{2}{*}{ Cardiovascular diseases } & \multicolumn{3}{|c|}{$<1$ year of follow-up } & \multicolumn{3}{|c|}{$\geq 1$ year of follow-up } \\
\hline & $\begin{array}{l}\text { No of cases in } \\
\text { patients/siblings }\end{array}$ & $\begin{array}{l}\text { Hazard ratio } \\
(95 \% \mathrm{Cl})\end{array}$ & $\begin{array}{l}\text { Hazard ratio } \\
(95 \% \mathrm{Cl})\end{array}$ & $\begin{array}{c}\text { No of cases in } \\
\text { patients/siblings }\end{array}$ & $\begin{array}{l}\text { Hazard ratio } \\
(95 \% \mathrm{Cl})\end{array}$ & $\begin{array}{l}\text { Hazard ratio } \\
(95 \% \mathrm{Cl})\end{array}$ \\
\hline Ischaemic heart disease, all & $161 / 193$ & $\rightarrow$ & 1.53 (1.18 to 2.00$)$ & $2121 / 3525$ & $=$ & $1.34(1.25$ to 1.44$)$ \\
\hline Acute myocardial infarction & $84 / 104$ & $\rightarrow$ & 1.37 (0.94 to 1.99$)$ & $1040 / 1796$ & - & 1.29 (1.17 to 1.43$)$ \\
\hline Other ischaemic heart disease & $77 / 89$ & $\rightarrow-$ & 1.77 (1.20 to 2.61$)$ & $1081 / 1729$ & ㄴ & $1.39(1.26$ to 1.53$)$ \\
\hline Cerebrovascular disease, all & $120 / 119$ & $\rightarrow$ & 1.75 (1.26 to 2.42$)$ & $1417 / 2006$ & - & $1.42(1.31$ to 1.55$)$ \\
\hline Arachnoidal bleeding & $12 / 7$ & & 2.81 (0.83 to 9.55$)$ & $139 / 206$ & $\rightarrow$ & 1.27 (0.97 to 1.65$)$ \\
\hline Haemorrhagic stroke & $11 / 24$ & 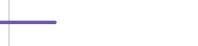 & $0.76(0.29$ to 1.96$)$ & $210 / 301$ & $\rightarrow$ & $1.56(1.24$ to 1.95$)$ \\
\hline Ischaemic stroke & $75 / 76$ & $\rightarrow-$ & 2.01 (1.28 to 3.14$)$ & $834 / 1201$ & - & 1.44 (1.29 to 1.61$)$ \\
\hline Other cerebrovascular disease & $22 / 12$ & $\longrightarrow$ & 5.64 (1.19 to 26.75$)$ & $234 / 298$ & $\rightarrow$ & 1.51 (1.21 to 1.89$)$ \\
\hline Emboli and thrombosis, all & $61 / 56$ & $\rightarrow-$ & 1.75 (1.09 to 2.80$)$ & $622 / 749$ & $=$ & $1.52(1.34$ to 1.74$)$ \\
\hline Artery thrombosis/embolus & $9 / 8$ & $\rightarrow$ & $1.37(0.21$ to 8.78$)$ & $110 / 117$ & $\rightarrow$ & 2.02 (1.45 to 2.82$)$ \\
\hline Pulmonary emboli & $52 / 48$ & $\longrightarrow$ & 1.75 (1.04 to 2.93$)$ & $512 / 632$ & $=$ & $1.45(1.26$ to 1.67$)$ \\
\hline Hypertensive diseases, all & $168 / 143$ & $\rightarrow$ & 2.15 (1.61 to 2.86$)$ & $1531 / 2567$ & - & $1.16(1.08$ to 1.26$)$ \\
\hline Essential hypertension & $151 / 129$ & $\rightarrow$ & 2.13 (1.57 to 2.90$)$ & $1407 / 2378$ & - & $1.16(1.07$ to 1.26$)$ \\
\hline Other hypertensive disease & $17 / 14$ & $\longrightarrow$ & 2.27 (0.80 to 6.45$)$ & $124 / 189$ & $\rightarrow$ & 1.28 (0.95 to 1.73$)$ \\
\hline Heart failure, all & $41 / 29$ & $\longrightarrow$ & $2.52(1.25$ to 5.08$)$ & $609 / 933$ & 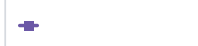 & $1.39(1.22$ to 1.60$)$ \\
\hline Heart failure & $32 / 15$ & $\longrightarrow$ & 6.95 (1.88 to 25.68$)$ & $498 / 763$ & $=$ & 1.39 (1.19 to 1.63$)$ \\
\hline Ischaemic cardiomyopathy & $1 / 1$ & & - & $22 / 41$ & $\rightarrow-$ & 1.50 (0.71 to 3.15$)$ \\
\hline Takotsubo cardiomyopathy & $8 / 13$ & & $0.78(0.28$ to 2.18$)$ & $89 / 129$ & $\rightarrow$ & 1.55 (1.12 to 2.14$)$ \\
\hline Arrhythmia/conduction disorder, all & $196 / 215$ & $\rightarrow$ & $1.36(1.08$ to 1.72$)$ & $1975 / 3222$ & - & $1.16(1.08$ to 1.24$)$ \\
\hline Arrhythmia & $146 / 186$ & $\rightarrow$ & 1.21 (0.93 to 1.57$)$ & $1592 / 2704$ & - & $1.12(1.04$ to 1.21$)$ \\
\hline Conduction disorder & $25 / 14$ & & 5.00 (1.58 to 15.80$)$ & $167 / 261$ & $\rightarrow$ & 1.17 (0.92 to 1.50$)$ \\
\hline Cardiac arrest & $25 / 15$ & $=$ & 3.37 (1.05 to 10.75$)$ & $216 / 257$ & $\rightarrow$ & $1.46(1.16$ to 1.85$)$ \\
\hline Fatal cardiovascular events, all & $96 / 66$ & $\longrightarrow-$ & $1.72(1.10$ to 2.68$)$ & $813 / 1030$ & $=$ & 1.56 (1.37 to 1.77$)$ \\
\hline
\end{tabular}

Fig 2 | Relative risks of developing different types of cardiovascular disease among patients with any stress related disorder, compared with their full siblings, by time of follow-up ( $<1$ or $\geq 1$ year). All Cox models were stratified by family identifiers and adjusted for age at index date, sex, educational level, family income, marital status, history of severe somatic diseases, and history of other psychiatric disorders. Time since the index date was used as underlying time scale

arrhythmia to 2.02 (1.45 to 2.82) for artery thrombosis/ embolus). Focusing on acute cardiovascular events only, we found a clear time dependent risk pattern for cardiac arrest, indicating a more than fourfold relative risk within the first six months of diagnosis of a stress related disorder (supplementary table G). The temporal risk patterns for acute myocardial infarction and acute cerebrovascular disease were similar but less prominent.

The presence of psychiatric comorbidity did not substantially modify the risk elevations for cardiovascular disease after a diagnosis of a stress related disorder, except for fatal cardiovascular events, for which the association was distinctly amplified by the presence of psychiatric comorbidity (supplementary figure B). In addition, we obtained almost identical results in the sensitivity analysis using a more conservative definition of "history of other psychiatric disorders” (supplementary table H).

\section{Discussion}

In this nationwide population based and sibling controlled study, we found that people with stress related disorders were at elevated risk of multiple types of cardiovascular disease, especially early onset ones (incident age $<50$ ). The relative risk elevation was independent of sex, familial background (including family history of cardiovascular disease), history of psychiatric/somatic diseases, and psychiatric comorbidity. Furthermore, the relative risks of severe and acute cardiovascular events (for example, cardiac arrest) were highest during the period adjacent to the diagnosis of a stress related disorder, indicating the first six months after diagnosis as a high risk time window. Likewise, the excess relative risks of other studied cardiovascular diseases were more pronounced during the first year after diagnosis of a stress related disorder than thereafter. Notably, although the highest point estimates were consistently noted for PTSD, other stress related disorders also conferred a considerably increased risk of cardiovascular disease.

\section{Strengths and weaknesses of study}

The merits of our study include the population based, sibling controlled design, which provides optimal conditions for relieving important concerns regarding genetic and early life environmental confounders, ${ }^{22}$ as well as pre-existing or co-occurring psychiatric disorders. The diagnoses of stress related disorders and cardiovascular disease were obtained prospectively and independently, minimising the risk of information bias. The cross linkages to the Cause of Death and Emigration Registers reduced selection bias due to loss of follow-up. Importantly, by involving more than 100000 patients 
with stress related disorders with up to 27 years of follow-up, the large sample size provided sufficient power to do most of the planned subgroup analyses. The availability of demographic, clinical, and family information enabled consideration of a wide range of health and disease related factors in the analysis.

Limitations include, firstly, the absence of information from primary care as well as late inclusion of outpatient specialist care records in the National Patient Register, which might have led to an underestimation of the number of patients with milder forms of stress related disorders or less severe cardiovascular diseases. Secondly, changes in the diagnostic criteria for stress related disorders over the 27 year study period may have influenced the observed associations, although our stratified analyses across different calendar periods suggest a minor effect of such diagnostic instability on the reported associations. Thirdly, as traumatic life experiences may be shared within families, a proportion of the reference (sibling) population may also have had milder or undiagnosed stress related disorders, yielding conservative estimates in the sibling analysis. Fourthly, as other psychiatric disorders both are risk factors for and frequently appear together with stress related disorders, distinguishing co-occurring from pre-existing psychiatric disorders in register based studies is challenging. However, such concern was partly alleviated by our sensitivity analyses in which introduction of an alternative definition for "history of other psychiatric disorders" yielded similar results. Fifthly, we had no data on behaviour related factors (such as smoking and alcohol consumption) that may contribute to the observed association as confounders or mediators. As these behavioural factors tend to cluster within families, our sibling analysis should have relieved the concern of residual confounding to some extent. However, further studies with detailed information on lifestyle, especially potential changes in lifestyle after diagnosis of stress related disorders, are warranted to clarify the impact of these factors on the association of interest. Finally, as this study focuses on patients who received a clinical diagnosis of stress related disorders through a hospital visit, its findings might not be directly applicable to people with less severe stress reactions or daily stress.

\section{Comparison with other studies}

Our findings corroborate the results from previous prospective studies suggesting a possible role of stress related disorders in the pathogenesis of cardiovascular disease. $^{11} 12$ 28-35 However, the main body of the preceding evidence was primarily derived from studies of male samples (veterans or active duty military personnel), ${ }^{12} 293035$ focusing almost exclusively on PTSD or self reported symptoms of PTSD. ${ }^{1130}$ Data on the role of stress related disorders in cardiovascular disease in women were, until now, limited. ${ }^{31-34}$ Although some attempts have been made to control for familial factors and co-occurring psychiatric disorders, ${ }^{12} 33$ or to study other stress related disorders (for example, adjustment disorder), ${ }^{34}$ no previous initiative has considered all these concerns in the same study. Also, the modest sample sizes of many previous studies often precluded analyses of different subtypes of cardiovascular disease. As a result, our study is the first to show a robust association between stress related disorders, including but not limited to PTSD, and multiple types of cardiovascular disease among both men and women, independent of familial factors and psychiatric comorbidities. Our findings indicate that the risk elevation generally exists for all studied cardiovascular diseases, with the strongest associations observed for the major categories of heart failure (within one year after the diagnosis of any stress related disorder) and emboli/thrombosis (beyond one year). These findings gain support from previous studies on 49000 US female nurses, showing a higher hazard ratio for venous thromboembolism by burden of PTSD symptoms compared with other cardiovascular disease outcomes, including myocardial infarction or stroke. ${ }^{32} 33$ Similarly, one longitudinal study indicated a positive association between PTSD and incident heart failure among veterans, although no risk estimate was provided for the first year after PTSD specifically. ${ }^{36}$

Clinical observations suggest that experiencing severe emotional or physical stress may trigger immediate cardiovascular consequences, such as heart attack and sudden cardiac arrest, even in apparently healthy people. ${ }^{37} 38$ Our results consolidate these reported associations by showing that a clinically confirmed stress related disorder (requiring a preceding occurrence of a trauma or significant life stressor) is also associated with these acute cardiovascular events.

\section{Meaning of study}

Although the crucial underlying mechanisms remain unclear, many potential mechanisms have been proposed to explain the association between stress related disorders, particularly PTSD, and cardiovascular disease. The physiological effects of an acute stressor can directly affect the cardiovascular system (for example, increased arterial blood pressure), which consequently forms favourable conditions for the onset of acute cardiovascular events, ${ }^{39} 40$ as well as the further development of hypertension, endothelial dysfunction, and arteriosclerosis. ${ }^{41}$ In addition, the long lasting effect of severe stress reactions on cardiovascular risk is also plausible through prolonged biological disturbances (for example, inflammation, autonomic dysfunction, dysregulation of hypothalamic-pituitary-adrenal axis, and abnormal neurochemistry $)^{42-44}$ and behaviour related changes (for example, smoking and sleep disturbance). ${ }^{33}$ A recent study in 4178 US veterans (aged 30-70 years) indicates that the association between PTSD and incident cardiovascular disease may be mediated by behavioural factors, metabolic conditions, and mental disorders. ${ }^{45}$ As our analyses showed that patients with stress related disorders other than PTSD were also at a considerably excess risk of adverse cardiovascular outcomes, further studies with 
a broader research scope are warranted to explore the underlying mechanisms between the various subtypes of stress related disorders and the development of cardiovascular disease.

One challenge related to this topic of study is how to assess the immediate effect of stress related disorders on cardiovascular disease. The main concerns include the risk of reverse causality (that is, cardiac symptoms emerge first and contribute to the diagnosis of stress related disorders) and surveillance bias (that is, patients with stress related disorders have more healthcare visits than others, leading to a higher likelihood of receiving a diagnosis of cardiovascular disease). In this study, despite the possibility that we might have missed the greatest risk increase (and some acute fatal outcomes) by following patients from their date of diagnosis of stress related disorders, instead of the date when they were exposed to the actual stressor or trauma, the time dependent hazard ratio for cardiovascular disease indicates that the first year, especially the first six months, after clinical diagnosis of stress related disorders entails the highest excess cardiovascular risk among the affected patients. This may be interpreted as a mixed consequence of the abovementioned factors and a real immediate effect of the stress reactions to trauma on cardiovascular disease. However, the additional analyses on acute and severe cardiovascular events (that is, cardiac arrest, acute myocardial infarction, and acute cerebrovascular disease) that typically result in prompt hospital visit and medical care provide further evidence supporting an immediate effect of stress related disorders on cardiovascular disease, as such analyses are less likely to be affected by surveillance bias or reverse causality. These sudden cardiovascular events carry a high risk of a fatal outcome, ${ }^{46}$ so increased clinical awareness of these risks among patients with recently diagnosed stress related disorders deserves further attention.

\section{Conclusions}

This population based, sibling controlled analysis showed a clear association between clinically confirmed stress related disorders and a higher subsequent risk of cardiovascular disease, particularly during the months after diagnosis of a stress related disorder, in the Swedish population. This association applies equally to men and women and is independent of familial factors, history of somatic/psychiatric diseases, and psychiatric comorbidities. These findings call for enhanced clinical awareness and, if verified, monitoring or early intervention among patients with recently diagnosed stress related disorders.

\footnotetext{
AUTHOR AFFILIATIONS

${ }^{1}$ Center of Public Health Sciences, Faculty of Medicine, University of Iceland, Reykjavík, Iceland

${ }^{2}$ Department of Medical Epidemiology and Biostatistics, Karolinska Institutet, Stockholm, Sweden

${ }^{3}$ National Centre for Disaster Psychiatry, Department of Neuroscience, Psychiatry, Uppsala University, Uppsala, Sweden ${ }^{4}$ Stress Research Institute, Stockholm University, Stockholm, Sweden
}

${ }^{5}$ Centre for Psychiatry Research, Department of Clinical Neuroscience, Karolinska Institutet, Stockholm, Sweden

${ }^{6}$ Stockholm Health Care Services, Stockholm County Council, Stockholm, Sweden

${ }^{7}$ Astrid Lindgren Children's Hospital, Karolinska University Hospital, Stockholm, Sweden

${ }^{8}$ Clinical Epidemiology and Biostatistics, School of Medical Sciences, Örebro University, Örebro, Sweden

${ }^{9}$ Department of Epidemiology, Harvard T H Chan School of Public Health, Boston, MA, USA

We thank Peter Green for his input on the readability and accuracy of this document during the review process of the study.

Contributors: UAV, FF, and HS were responsible for the study concept and design. HS, UV, and FF did the data analysis. UAV, HS, FF, GT, FKA $\mathrm{PL}, \mathrm{CA}, \mathrm{DMC}$, LFC, and KF interpreted the data. HS, UAV, FF, FKA, PL, CA, DMC, LFC, KF, and GT drafted the manuscript. HS and UAV had full access to all the data in the study and take responsibility for the integrity of the data and the accuracy of the data. The corresponding author attests that all listed authors meet authorship criteria and that no others meeting the criteria have been omitted. HS and UAV are the guarantors.

Funding: The study was supported by Grant of Excellence, Icelandic Research Fund (grant No 163362-051 to UAV), and ERC Consolidator Grant (StressGene, grant No 726413 to UAV); by the Karolinska Institutet (Senior Researcher Award and Strategic Research Area in Epidemiology to FF); and by the Swedish Research Council through the Swedish Initiative for Research on Microdata in the Social And Medical Sciences (SIMSAM) framework (grant No 340-2013-5867 to CA).

Competing interests: All authors have completed the ICMJE uniform disclosure form at www.icmje.org/coi_disclosure.pdf (available on request from the corresponding author) and declare: no support from any organisation for the submitted work other than that detailed above; no financial relationships with any organisations that might have an interest in the submitted work in the previous three years; no other relationships or activities that could appear to have influenced the submitted work.

Ethical approval: The study was approved by the Regional Ethics Review Board in Stockholm, Sweden (Dnr 2013/862-31/5). Data sharing: No additional data available.

Transparency: The study guarantors affirm that this manuscript is an honest, accurate, and transparent account of the study being reported; that no important aspects of the study have been omitted; and that any discrepancies from the study as planned (and, if relevant, registered) have been explained.

This is an Open Access article distributed in accordance with the Creative Commons Attribution Non Commercial (CC BY-NC 4.0) license, which permits others to distribute, remix, adapt, build upon this work non-commercially, and license their derivative works on different terms, provided the original work is properly cited and the use is noncommercial. See: http://creativecommons.org/licenses/by-nc/4.0/.

1 Kessler RC, Aguilar-Gaxiola S, Alonso J, et al. Trauma and PTSD in the WHO World Mental Health Surveys. Eur I Psychotraumatol 2017;8(Suppl 5):1353383. doi:10.1080/200081 98.2017.1353383

2 de Vries GJ, Olff M. The lifetime prevalence of traumatic events and posttraumatic stress disorder in the Netherlands. / Trauma Stress 2009;22:259-67. doi:10.1002/jts.20429

3 Fall K, Fang F, Mucci LA, et al. Immediate risk for cardiovascular events and suicide following a prostate cancer diagnosis: prospective cohort study. PLoS Med 2009;6:e1000197. doi:10.1371/journal. pmed.1000197

4 Shen Q, Lu D, Schelin ME, et al. Injuries before and after diagnosis of cancer: nationwide register based study. BMJ 2016;354:14218. doi:10.1136/bmj.i4218

5 Cohen S, Tyrrell DA, Smith AP. Psychological stress and susceptibility to the common cold. N Engl / Med 1991:325:606-12. doi:10.1056/ NEJM199108293250903

6 Song H, Fang F, Tomasson G, et al. Association of StressRelated Disorders With Subsequent Autoimmune Disease. JAMA 2018;319:2388-400. doi:10.1001/jama.2018.7028

7 Bergelt C, Prescott E, Grønbaek M, Koch U, Johansen C. Stressful life events and cancer risk. Br J Cancer 2006;95:1579-81. doi:10.1038/ sj.bjc.6603471

8 Gradus JL, Farkas DK, Svensson E, et al. Posttraumatic stress disorder and cancer risk: a nationwide cohort study. Eur Epidemiol 2015;30:563-8. doi:10.1007/s10654-015-0032-7 
9 Gradus JL, Antonsen S, Svensson E, Lash TL, Resick PA, Hansen JG. Trauma, comorbidity, and mortality following diagnoses of severe stress and adjustment disorders: a nationwide cohort study. Am J Epidemiol 2015;182:451-8. doi:10.1093/aje/kwv066

10 Li J, Precht DH, Mortensen PB, Olsen J. Mortality in parents after death of a child in Denmark: a nationwide follow-up study. Lancet 2003;361:363-7. doi:10.1016/S0140-6736(03)12387-2

11 Jordan HT, Stellman SD, Morabia A, et al. Cardiovascular disease hospitalizations in relation to exposure to the September 11, 2001 World Trade Center disaster and posttraumatic stress disorder. J Am Heart Assoc 2013;2:e000431. doi:10.1161/JAHA.113.000431

12 Vaccarino V, Goldberg J, Rooks C, et al. Post-traumatic stress disorder and incidence of coronary heart disease: a twin study. J Am Coll Cardiol 2013;62:970-8. doi:10.1016/j.jacc.2013.04.085

13 O'Donovan A, Cohen BE, Seal KH, et al, Elevated risk for autoimmune disorders in iraq and afghanistan veterans with posttraumatic stress disorder. Biol Psychiatry 2015;77:365-74. doi:10.1016/j. biopsych.2014.06.015

14 American Psychiatric Association. Diagnosis and startistical manual of medical disorders. 4th ed. APA, 1994

15 World Health Organization. The ICD-10 classification of mental and behavioral disorders: Clinical description and diagnostic guidelines. WHO, 1992.

16 Ursano RJ, Bell C, Eth S, et al, Work Group on ASD and PTSD, Steering Committee on Practice Guidelines. Practice guideline for the treatment of patients with acute stress disorder and posttraumatic stress disorder. Am J Psychiatry 2004;161(Suppl):3-31.

17 Auer PL, Stitziel NO. Genetic association studies in cardiovascular diseases: Do we have enough power?Trends Cardiovasc Med 2017:27:397-404. doi:10.1016/j.tcm.2017.03.005

18 Khera AV, Emdin CA, Drake I, et al. Genetic Risk, Adherence to a Healthy Lifestyle, and Coronary Disease. $N$ Engl Med 2016:375:2349-58 doi:10.1056/NEIMoa1605086

19 Norhammar A, Mellbin L, Cosentino F. Diabetes: Prevalence, prognosis and management of a potent cardiovascular risk factor. Eur / Prev Cardiol 2017:24(Suppl):52-60. doi:10.1177/2047487317709554

20 Ludvigsson JF, Otterblad-Olausson P, Pettersson BU, Ekbom A. The Swedish personal identity number: possibilities and pitfalls in healthcare and medical research. Eur J Epidemiol 2009;24:659-67. doi:10.1007/s10654-009-9350-y

21 National Collaborating Centre for Mental Health (UK). Post-Traumatic Stress Disorder: The Management of PTSD in Adults and Children in Primary and Secondary Care. Gaskell, 2005

22 D’Onofrio BM, Lahey BB, Turkheimer E, Lichtenstein P. Critical need for family-based, quasi-experimental designs in integrating genetic and social science research. Am J Public Health 2013;103(Suppl 1):S46-55. doi:10.2105/AJPH.2013.301252

23 Harvey AG, Bryant RA. The relationship between acute stress disorder and posttraumatic stress disorder: a 2-year prospective evaluation. J Consult Clin Psychol 1999;67:985-8. doi:10.1037/0022 006X.67.6.985

24 World Health Organization. Cardiovascular diseases (CVDs). 2017. http://www.who.int/mediacentre/factsheets/fs317/en/.

25 Gros DF, Price M, Magruder KM, Frueh BC. Symptom overlap in posttraumatic stress disorder and major depression. Psychiatry Res 2012;196:267-70. doi:10.1016/j.psychres.2011.10.022

26 Charlson ME, Pompei P, Ales KL, MacKenzie CR. A new method of classifying prognostic comorbidity in longitudinal studies: development and validation. / Chronic Dis 1987:40:373-83. doi:10.1016/0021-9681(87)90171-8

27 Lambert PC, Royston P. Further development of flexible parametric models for survival analysis. Stata / 2009:9:265-90. doi:10.1177/1536867X0900900206

28 Jordan HT, Miller-Archie SA, Cone JE, Morabia A, Stellman SD. Heart disease among adults exposed to the September 11 . 2001 World Trade Center disaster: results from the World Trade Center Health Registry. Prev Med 2011;53:370-6. doi:10.1016/j. ypmed.2011.10.014
29 Boscarino JA. A prospective study of PTSD and early-age heart disease mortality among Vietnam veterans: implications for surveillance and prevention. Psychosom Med 2008;70:668-76. doi:10.1097/PSY.0b013e31817bccaf

30 Kubzansky LD, Koenen KC, Spiro A3rd, Vokonas PS, Sparrow D. Prospective study of posttraumatic stress disorder symptoms and coronary heart disease in the Normative Aging Study. Arch Gen Psychiatry 2007;64:109-16. doi:10.1001/archpsyc.64.1.109

31 Kubzansky LD, Koenen KC, Jones C, Eaton WW. A prospective study of posttraumatic stress disorder symptoms and coronary heart disease in women. Health Psychol 2009;28:125-30. doi:10.1037/02786133.28.1.125

32 Sumner JA, Kubzansky LD, Elkind MSV, et al. Trauma Exposure and Posttraumatic Stress Disorder Symptoms Predict Onset of Cardiovascular Events in Women. Circulation 2015;132:251-9. doi:10.1161/CIRCULATIONAHA.114.014492

33 Sumner JA, Kubzansky LD, Kabrhel C, et al. Associations of Trauma Exposure and Posttraumatic Stress Symptoms With Venous Thromboembolism Over 22 Years in Women. J Am Heart Assoc 2016:5:e003197. doi:10.1161/JAHA.116.003197

34 Gradus JL, Farkas DK, Svensson E, et al. Associations between stress disorders and cardiovascular disease events in the Danish population. BMJ Open 2015;5:e009334. doi:10.1136/ bmjopen-2015-009334

35 Burg MM, Brandt C, Buta E, et al. Risk for Incident Hypertension Associated With Posttraumatic Stress Disorder in Military Veterans and the Effect of Posttraumatic Stress Disorder Treatment. Psychosom Med 2017;79:181-8.

36 Roy SS, Foraker RE, Girton RA, Mansfield AJ. Posttraumatic stress disorder and incident heart failure among a community-based sample of US veterans. Am J Public Health 2015;105:757-63. doi:10.2105/AJPH.2014.302342

37 Ziegelstein RC. Acute emotional stress and cardiac arrhythmias. JAMA 2007;298:324-9. doi:10.1001/jama.298.3.324

38 Connelly KA, Maclsaac Al, Jelinek VM. Stress, myocardial infarction, and the "tako-tsubo" phenomenon. Heart 2004:90:e52. doi:10.1136/hrt.2004.038851

39 Rosengren A, Hawken S, Ounpuu S, et al, INTERHEART investigators. Association of psychosocial risk factors with risk of acute myocardial infarction in 11119 cases and 13648 controls from 52 countries (the INTERHEART study): case-control study. Lancet 2004;364:95362. doi:10.1016/S0140-6736(04)17019-0

40 Sharkey SW, Lesser JR, Zenovich AG, et al. Acute and reversible cardiomyopathy provoked by stress in women from the United States. Circulation 2005;111:472-9. doi:10.1161/01. CIR.0000153801.51470.EB

41 Steptoe A, Kivimäki M. Stress and cardiovascular disease. Nat Rev Cardiol 2012;9:360-70. doi:10.1038/nrcardio.2012.45

42 Koenen KC, Sumner JA, Gilsanz P, et al. Post-traumatic stress disorder and cardiometabolic disease: improving causal inference to inform practice. Psychol Med 2017;47:209-25. doi:10.1017/ S0033291716002294

43 Wentworth BA, Stein MB, Redwine LS, et al. Post-traumatic stress disorder: a fast track to premature cardiovascular disease?Cardiol Rev 2013;21:16-22. doi:10.1097/CRD.0b013e318265343b

44 Cohen S, Janicki-Deverts D, Doyle WJ, et al. Chronic stress, glucocorticoid receptor resistance, inflammation, and disease risk. Proc Natl Acad Sci U S A 2012;109:5995-9. doi:10.1073/ pnas.1118355109

45 Scherrer JF, Salas J, Cohen BE, et al. Comorbid Conditions Explain the Association Between Posttraumatic Stress Disorder and Incident Cardiovascular Disease. J Am Heart Assoc 2019;8:e011133. doi:10.1161/JAHA.118.011133

46 Engdahl J, Holmberg M, Karlson BW, Luepker R, Herlitz J. The epidemiology of out-of-hospital 'sudden' cardiac arrest. Resuscitation 2002;52:235-45. doi:10.1016/S0300 9572(01)00464-6

\section{Supplementary materials}

